\title{
Planning Considerations for a Mars Sample Receiving Facility: Summary and Interpretation of Three Design Studies
}

\author{
David W. Beaty, Carlton C. Allen, ${ }^{2}$ Deborah S. Bass,, Karen L. Buxbaum, James K. Campbell,,* \\ David J. Lindstrom, ${ }^{3}$ Sylvia L. Miller, ${ }^{1, *}$ and Dimitri A. Papanastassiou ${ }^{4}$
}

\begin{abstract}
It has been widely understood for many years that an essential component of a Mars Sample Return mission is a Sample Receiving Facility (SRF). The purpose of such a facility would be to take delivery of the flight hardware that lands on Earth, open the spacecraft and extract the sample container and samples, and conduct an agreedupon test protocol, while ensuring strict containment and contamination control of the samples while in the SRF. Any samples that are found to be non-hazardous (or are rendered non-hazardous by sterilization) would then be transferred to long-term curation. Although the general concept of an SRF is relatively straightforward, there has been considerable discussion about implementation planning.

The Mars Exploration Program carried out an analysis of the attributes of an SRF to establish its scope, including minimum size and functionality, budgetary requirements (capital cost, operating costs, cost profile), and development schedule. The approach was to arrange for three independent design studies, each led by an architectural design firm, and compare the results. While there were many design elements in common identified by each study team, there were significant differences in the way human operators were to interact with the systems. In aggregate, the design studies provided insight into the attributes of a future SRF and the complex factors to consider for future programmatic planning. Key Words: Mars-Sample Receiving Facility (SRF)— Mars Sample Return (MSR)—Curation-Biosafety-Test protocol—Sample preservation-Containment-Clean room-NASA—Planetary protection. Astrobiology 9, 745-758.
\end{abstract}

\section{Introduction}

A Rовотіс Mission to collect samples of Mars and transport them to Earth has been considered in one form or another for more than three decades (e.g., NRC, 1978, 1990a, 1990b, 1994, 1996, 2002, 2007; MEPAG ND-SAG, 2008, and references therein). Although different variants of this mission over the years have taken different names, we refer to the mission described in this paper as Mars Sample Return, or MSR. In an engineering sense, MSR as a flight mission is one of the most complex undertakings NASA and its European partners have ever considered-there are some fascinating challenges related to the flight system (see, e.g., Bar-Cohen et al., 2005; Gershman et al., 2005; Mattingly et al., 2005; Stephenson and Willenberg, 2006; iMARS, 2008; Moura et al., 2008; Backes et al., 2009). In addition to the complexities of the flight system, the planning for management of the samples once they arrive on Earth is equally critical. Perhaps the most important single element of the "ground system" is a facility referred to as the Sample Receiving Facility (SRF), whose purpose would be to receive the returned spacecraft, extract the sealed sample container, open it to access the samples, and then carry out a set of tests under strict containment conditions to determine whether the samples are hazardous.

The SRF element of the overall mission is mandatory because of international planetary protection agreements (see, e.g., Atlas, 2008; COSPAR, 2008). The NRC (1997) pointed out that, though the probability of extant martian life in such a returned sample is very low, it is nonzero. Because of this, a sample returned from Mars would be subject to the very rigorous rules and practices in place to protect Earth from the potential risk of extraterrestrial life. The interested reader can

\footnotetext{
${ }^{1}$ Mars Program Office, and ${ }^{4}$ Science Division, Jet Propulsion Laboratory, California Institute of Technology, Pasadena, California

${ }^{2}$ NASA Johnson Space Center, Houston, Texas.

${ }^{3}$ NASA Headquarters, Washington, DC.

${ }^{*}$ Retired, Mars Program Office, Jet Propulsion Laboratory, California Institute of Technology, Pasadena, California.
} 
refer to the specifics of these policies at COSPAR (2008) and MEPAG ND-SAG $(2005,2008)$. Although the purpose of the policy is clear, there has been considerable debate about the nature of the facility required to implement it.

The purpose of this paper is to summarize a set of studies that were undertaken in 2003 to help constrain the minimum facility for policy compliance, with a specific goal of defining its probable basic attributes and estimating its cost. Although MSR is not currently approved by any of the international space agencies, this information will be needed prior to planning the budget and timeline for the SRF should MSR proceed in the future.

\section{A. History and context}

The first in-depth discussion of facility planning for an MSR-related SRF was carried out in connection with planning for the MSR 2003-2005 Project. The term "Mars Receiving Facility" was introduced at the Mars Sample Handling, Distribution, and Analysis Workshop (D. McCleese and M. Drake, Chairs), which was held at Caltech in February 1999. This facility concept was renamed later that year, however, to the more generic "Sample Receiving Facility" (or SRF) by NASA's newly formed Mars Returned Sample Handling team because of the possibility that such a facility might be used in the future for samples originating from planetary objects other than Mars.

Reports from studies and workshops during the decade leading up to the 2003-2005 MSR effort established the context for SRF design and implementation. In 1995, NASA asked the National Research Council (NRC) to conduct a study pertaining to sample return and address the key issues ${ }^{1}$ associated with the potential risks to Earth of samples returned from Solar System bodies, such as Mars. The NRC panel focused principally on Mars and produced a report (NRC, 1997) that became a cornerstone for much of the planetary protectionrelated work of the MSR 2003-2005 Project. In addition, the NASA Mars Sample Handling and Requirements Panel (MSHARP) made recommendations (Carr et al., 1999) regarding what is required to certify returned samples as nonhazardous and the considerations associated with sample receiving, curation, and distribution. MSHARP recommended that the samples be treated as hazardous until proven otherwise, consistent with the NRC (1997).

Because the earlier reports provided advice at a fairly general level, the NRC followed up with a study of the criteria for release of samples from biocontainment in an SRF (NRC, 2002). Of relevance to the present study, the report recommended that only the most basic operations should be conducted inside the facility, and it should be designed to the smallest and simplest possible scale consistent with its dual

\footnotetext{
${ }^{1}$ The NRC (1997) addressed the following issues: (a) the potential and probability for a living entity to be included in a sample returned from another Solar System body, in particular Mars; (b) the scientific investigations that should be conducted to reduce uncertainty in the above assessment; (c) the potential for large-scale effects on the environment resulting from the release of any returned entity (d) the status of technological measures that could be taken on a mission to prevent the unintended release of a returned sample into Earth's biosphere; and (e) the criteria for controlled distribution of sample material, taking note of the anticipated regulatory framework.
}

roles as a biological containment and clean-room facility, with detailed protocols and procedures for handling and testing martian samples. This report additionally argued that it would take at least 7 years in advance of the anticipated return of martian samples to plan and construct an SRF.

Beginning in 1997, NASA sponsored a series of workshops (e.g., DeVincenzi et al., 1999; Race and Rummel, 2000; Race et al., 2001a, 2001b) to prepare a first draft of the test protocol that would evaluate the safety of returned martian samples. The overall objective was "to produce a draft protocol by which returned martian sample materials could be assessed for biological hazards and examined for evidence of life (extant or extinct), while safeguarding the purity of the samples from possible terrestrial contamination." The resulting Draft Test Protocol (Rummel et al., 2002) was published with the expectation that there would be continued revisions as more information became available about Mars and analytical techniques improved. The Draft Test Protocol is described in greater detail below, because it served as a source of design requirements for the SRF studies summarized here.

NASA's MSR 2003-2005 Project advanced as far as its Preliminary Design Review before it was cancelled in 2000 (O'Neil and Cazaux, 2000). NASA recognized, however, that if an MSR project were restarted in the future, it must include realistic planning parameters for the SRF, including cost, schedule, and size. Therefore, planning activity for the SRF continued through 2004, several years beyond the cancellation of the flight mission. A major aspect of this planning was a set of three independent competed industry studies that were carried out in 2003-2004. These studies have formed the basis for a much clearer understanding of the possible requirements, design, cost, timeline, and operational considerations for an MSR-related SRF and are the focus of this paper.

\section{Methods and Conceptual Requirements}

No individual facilities currently exist that meet all the requirements of the SRF (which are described in a following section) (see also Atlas, 2008). Moreover, there are technical reasons related to achievement of the necessary standards of cleanliness, which led us to conclude that it was very unlikely to achieve an acceptable SRF by modifying an existing building. Thus, for the purpose of this study, we focused only on the planning of a new facility. This would constitute a reference point to which possible alternative options related to modification of existing facilities could be compared in the future.

In response to a NASA-originated solicitation, seven letters of interest were received in May 2003. Through an evaluation board, three architectural design teams were selected to carry out separate $\$ 200$ thousand, 6-month studies. Each team had industry experts who specialize in cleanroom design, biohazard considerations, aseptic processing, robotics, and advanced instrumentation. The three teams were led by Industrial Design and Construction (IDC) of Portland, Oregon; Lord, Aeck, Sargent (LAS) of Atlanta, Georgia; Flad \& Associates (FLAD) of Madison, Wisconsin. The three industry teams operated independently and delivered their analyses in 2004 in the form of final reports to be used by NASA for future planning.

Each of the architectural design teams was asked to conduct a design study and also provide a cost estimate for an 
SRF that operates at a containment level equivalent to Biosafety Level 4 (BSL-4). For the purpose of the analysis, it was assumed that the SRF would receive samples from Mars collected by a mission launched in 2013, with the samples returned to Earth at the end of 2016, by which time the SRF would have been certified to receive samples. The teams were asked to prepare designs that would meet requirements for (a) ensuring containment of potential nonterrestrial biological material in the sample, (b) preventing contamination of the sample by terrestrial contaminants (biological and inorganic materials), and (c) permitting preliminary examination, hazard assessment, and life-detection analyses of the samples.

\section{A. Draft test protocol}

The design teams were told that the SRF concepts must permit implementation of the Draft Test Protocol (Rummel et al., 2002). This document lays out an approach for returned samples to be subsampled and subjected to "sufficient testing to evaluate them against the release criteria." The Protocol itself has three main segments: physical/chemical processing, life-detection testing, and biohazard testing. In the Protocol, these tests are defined, and flow charts are used to complement the text in describing the conceptual flow through the test process. There is emphasis throughout on approaches to sample examination that are credible, thorough, and informative while maintaining a priority for sample preservation-both in quantity and quality. The developers of the Protocol were careful to point out that the tests must be sufficient to answer the key questions concerning possible life and biohazard while preserving, to the greatest extent possible, the quantity of sample available for future science. Therefore, the Draft Test Protocol "attempts a compromise between the desire to destructively analyze only a small proportion of the returned sample by planetary protection testing, and the need to assure safety by testing all portions of all samples." All the while, the sample portions preserved for science must remain pure (uncontaminated) and unaltered to the greatest extent possible.

\section{B. Facility scope}

For the purpose of the industry studies, long-term, major scientific research in the SRF was not to be consideredeither the samples meet the release criteria and are allocated to non-containment laboratories or additional facilities at the SRF must be created. The design teams were told that SRF concepts under consideration should represent the minimum facility to implement the Draft Test Protocol on representative samples within 6-9 months of sample receipt. To comply with the Draft Test Protocol, the teams were to assume the need for testing on live animals (although it was recognized that this need might eventually be eliminated). Further, they were to address whether it would be preferable to include this functionality in the SRF or make use of some secondary facility that meets the SRF containment requirements though not necessarily the sample cleanliness requirements.

The design concepts for the SRF had to provide for the following:

(1) Receiving and opening the spacecraft, removing the samples;
(2) Sample splitting and packaging as required to provide subsamples for further testing as specified in the Draft Test Protocol;

(3) The capability to sterilize subsamples for analysis outside of containment;

(4) The sterilization of any waste products from the SRF that might have been exposed to martian samples;

(5) The capability to prepare and package martian samples that have been certified non-hazardous for transfer to a Mars Sample Curation Facility and distribution for further science investigations.

\section{C. Containment and sample purity}

The special considerations of biocontainment, coupled with the limited amount of precious sample and the scientific requirements for sample purity, were fundamental to the industry concept studies. For the purposes of the studies, contamination of returned martian samples fell into three broad categories, based on expected and proposed areas of scientific investigations: biological, organic, and inorganic. In practice, an investigation in one of these areas might not be adversely affected by contamination in a different area. However, the first-order principle was that all three categories must be considered together. A report by Neal (2000) on behalf of the NASA Curation and Analysis Planning Team for Extraterrestrial Materials (CAPTEM) addresses these issues in some detail. CAPTEM's recommendations, like those of the NRC (2002), recognized a major technical obstacle to designing the SRF: the problem of combining high-level biological containment with clean-room conditions. For example, when considering design of the containment facility for potentially hazardous samples, it is expected that samples would be stored under negative air pressure with respect to the outside laboratory to maximize biocontainment. By contrast, non-hazardous samples that must be kept pristine are typically stored under positive air pressure to minimize contamination. This illustrates the unusual requirement for an SRF to maintain both sample containment and cleanliness.

A significant part of the scientific purpose of MSR would be to evaluate returned martian samples for evidence of martian prebiotic chemistry, martian life itself, or both. Since Earth-sourced contaminants, particularly organic molecules, can give a false-positive signal, contamination control would be an essential aspect of the mission. It would be essential to sterilize and clean all elements that the samples contact between their native state on Mars and their analysis in instruments on Earth. An implication for the SRF is that the interior of the isolator cabinets, the trays, the sample containers, the sample-contact tools, and the sample chambers of the organic detection instruments would need to be cleaned to the same standards as for the sample-contact surfaces of the flight system. The standards and protocols for achieving this would have to be established, but this was not an assumption or SRF requirement for the study teams.

\section{D. US site selection issues}

If it is decided to proceed with selection of a location for an SRF, several issues would need to be addressed. First, one of two general selection processes would have to be chosen: 
either an internal down-select process or an external nomination process. The former would entail that a national agency, such as NASA, progressively narrow down on a site that meets its needs; the latter would involve a competition among candidate sites proposed by advocates. Although there are advantages and disadvantages to each approach, it is particularly worth noting that the competitive process would take significantly more time, and this would need to be taken into account in the planning and possibly costing. Second, any pass/fail threshold site criteria would need to be established. These might include perimeter security, government land ownership, access to transportation and utilities, avoidance of legally protected areas, and so on. Third, a set of evaluation criteria would need to be established to determine relative prioritization of candidate sites that pass the threshold criteria. In the US, it is required by the National Environmental Policy Act (NEPA) that the potential environmental impacts be considered early in the decision-making process, but other additional criteria might include fewest land use conflicts, user accessibility and convenience, risk due to natural hazards, and cost (including the cost of mitigating natural hazards). Finally, partnering options (of many different types) might play a significant role in improving the cost, efficiency, or management of the SRF. For example, placing the SRF adjacent to an existing BSL-4 lab might result in certain efficiencies, and this would clearly have site selection implications.

\section{E. Costing guidelines}

To prepare a cost estimate, the teams were given the following additional assumptions:

(a) The launch of the MSR mission was to occur in November 2013, and the assumed arrival of the sample back on Earth was July 2016.

(b) Cost estimates for the analytical capabilities, both acquisition and implementation, had to include multispectral imaging, microscopy, mass comparison, radiation counting, gas chromatography-mass spectrometry, Raman spectroscopy, X-ray diffraction, X-ray tomography, $\mathrm{X}$-ray fluorescence, atomic force microscopy, and scanning electron microscopy.

(c) No land-acquisition costs were included. The site for the SRF is treated as a vacant lot in an urban area. That is, roads, power, water and sewer are accessible.

(d) All tasks associated with NASA personnel were to be budgeted separately.

(e) All costs related to the external science community were to be budgeted separately.

\section{Existing Facility Analogues for SRF Functionalities}

As discussed above, there is no single facility in the world that would meet all the assumed requirements of the SRF. However, there are separate important facility engineering analogues for most of the aspects of the SRF, and in many respects the essence of the problem is in integrating these different aspects in a way that has never been done before.

\section{A. Astromaterials Curation Laboratories, Houston, TX}

An important model for the facilities issues associated with sample preservation is the Astromaterials Curation
Laboratories at NASA's Johnson Space Center. This complex is organized into a set of curation laboratories within two interconnected buildings. Thus, they share certain facility infrastructure (air, power, water), technical workforce, management, and perimeter security. The different sample collections (lunar rocks and soil, meteorites, cosmic dust, comet dust, solar wind atoms) are curated under different conditions, which are appropriate to the nature of the samples and the nature of the scientific questions these samples are being used to address. These labs are equipped to process, prepare, and distribute samples cleanly to science investigators across the globe.

The curation facility that is most relevant to returned martian samples is the Lunar Sample Laboratory, where the Apollo lunar samples are curated. These samples are stored in positive pressure, high-purity nitrogen gloveboxes within class 1,000 clean rooms. Concentrations of water vapor and oxygen in the gloveboxes are maintained at or below $50 \mathrm{ppm}$ and $20 \mathrm{ppm}$, respectively. The only materials to come into contact with the samples during processing are Teflon and precision-cleaned stainless steel and aluminum. These materials and procedures were developed to minimize inorganic and particulate contamination of the lunar rocks and soils.

\section{B. Biosafety Level 4 laboratories}

An alternative approach to evaluate requirements and key parameters for SRF planning was to consider analogous aspects of the planning, construction, and certification histories of BSL-4 laboratories (CDC/NIH, 2007; U.S. Department of Health and Human Services, 2007). As of 2004, there were six such labs or lab complexes in North America (Canadian Science Centre, Winnipeg, Canada; Centers for Disease Control and Prevention, Atlanta, Georgia; Georgia State University, Atlanta, Georgia; Southwest Research Institute, San Antonio, Texas; United States Army Medical Research Institute for Infectious Diseases, Ft. Detrick, Frederick, Maryland; and the University of Texas Medical Branch, Galveston, Texas), several of which had either recently been built or had recently added new buildings. Experience with these six facilities showed that the time from planning to certification in 2004 averaged about 8 years, though there was significant dispersion about this mean. However, BSL-4 laboratories do not have a requirement to keep their samples in pristine condition, so the relevance of the BSL-4 analogy was open to questions (Race and Hammond, 2008).

\section{C. Lunar Receiving Laboratory (LRL)}

An important precedent for SRF planning was the development of an analogous facility to support the return of lunar samples by the Apollo Program beginning in 1969. While in containment at the LRL, the samples were evaluated with a test protocol that had been developed in meetings at the Baylor College of Medicine in Houston; hence the name "Baylor Protocol" (Allton et al., 1998). The LRL and the Baylor Protocol were used for the Apollo 11, 12, and 14 missions, after which testing for biological hazards in the lunar samples was deemed unnecessary and was terminated.

There are several significant differences between the LRL and a future SRF. First, the amount of material to be returned by a MSR mission is assumed to be about $0.5 \mathrm{~kg}$ (iMARS, 2008; MEPAG ND-SAG, 2008), as compared to hundreds of 
kilograms returned by Apollo. Samples from six of the Apollo missions passed through the LRL, but only those from the first three missions did so under biological containment conditions. Second, the LRL was designed to quarantine the astronauts, their capsule, and the lunar rock and regolith samples, whereas the SRF would be designed for sample containment and contamination control only. (Sample containment refers to the protection of humans and the environment from sample hazards, whereas contamination control refers to the protection of sample purity.) Third, the process for securing approvals for construction of facilities has changed significantly. The LRL was completed in five years: initial planning for the LRL began in 1964, construction took place from 1966-1967, operational tests began in 1968, and certification was completed in early 1969 (Mangus and Larsen, 2004). A timeline this rapid is inconceivable today, primarily due to the passage of NEPA, which was signed into law on January 1, 1970. NEPA requires federal agencies to evaluate their proposed projects for potential environmental impacts, and the public must be allowed to comment on the evaluation. Though federal agency evaluations and subsequent public comment are time-consuming endeavors, they are to be considered in assessment of project alternatives prior to NASA taking steps to commit to an irreversible path.

\section{SRF Concept Results}

The design concepts submitted by the industry teams serve to highlight similarities and differences in how an SRF design might be approached in the future as well as key areas in which technology gaps would need to be filled.

\section{A. Primary functional attributes of an SRF}

There is a set of attributes that all three SRF concepts have in common. Although the teams made different implementation choices, each design includes the following:

(1) Receiving room to handle all Earth-return flight hardware. The Earth entry flight element of MSR would deliver the martian samples to Earth's surface with the sample canister intact. Regardless of what landing site is selected, the SRF designs assume that terrestrial organisms would contaminate the exterior of the return spacecraft. The SRF would need to be able to receive all the returned flight hardware and have a means by which to extract the samples from within the spacecraft containment systems without incurring contamination by abundant terrestrial life and without alteration of the samples.

(2) Secondary containment barrier. The SRF concepts all would provide a secondary containment barrier equivalent to structures found in BSL-4 laboratory complexes to back up the function of primary containment barriers. Space within this barrier is typically separated from the outside environment by an airlock and a sterilization system such as a chemical shower.

(3) Primary containment barrier. Within the containment facility, there would be isolation cabinets, within which a higher level of cleanliness could be maintained. In each design, these cabinets would provide the primary barrier between the sample and the envi- ronment, but the implementation details vary considerably (e.g., number, connectivity, approach to sample handling) as described below.

(4) Clean spaces for activities involving the pristine samples. All SRF designs would provide spaces within the primary containment barrier(s) where five different functions could be performed. These spaces would have the common attribute of being as close to pristine as possible to preserve sample purity and assure integrity of sample assessments. The necessary tools and instrumentation would be accommodated, though the approaches do vary. For example, instrumentation might be inside cabinets or on laboratory benches, depending on the specific approach to the containment barriers and sample manipulation. The five main functions would be:

(a) Initial sample characterization

(b) Pristine sample storage

(c) Space and tools for subdividing the samples

(d) Instruments and space needed for life detection

(e) Instruments and space needed for hazard assessment

(5) Means of moving samples and subsamples between functional elements. All SRF designs address the need to move samples and subsamples between pristine storage and various test or processing stations while maintaining containment and cleanliness standards. The SRF must also provide systems for detailed record keeping-essentially an early phase of sample curation.

(6) Air-handling system. One of the fundamental principles of biosafety is to have a system of air pressure gradients, so that potentially hazardous samples would be at a low air pressure relative to the human operators and the external environment. If something should leak, air flows toward, rather than away, from the potential hazard. Therefore, a major facility subsystem would be the air-handling capability.

(7) Waste sterilization system. It must be possible to sterilize solid and liquid waste products from the containment lab prior to release into the external environment.

(8) Capability to engage observers outside the containment lab both locally and remotely. There would be intense scientific and public interest in the returned martian samples. Given that the SRF would be engineered for minimal size and cost, only a limited number of people would be able to work in the containment labs. All teams concluded that it would be desirable to provide for an expanded set of people outside the containment barrier who have two-way communication in real time with the scientific and technical staff who are physically carrying out the various tests and other actions within the containment lab. There might be a need to accommodate scientists outside the barrier who would be both local (i.e., at the facility) and remote (i.e., at their home institutions).

(9) Office space for permanent staff, including management, research, and administrative. The SRF would need to provide for sufficient office space for the permanent staff.

(10) Office space for guest staff. Offices and conference rooms would need to be provided for outside researchers. This would be particularly important during the time martian samples are under primary evaluation in the facility. 


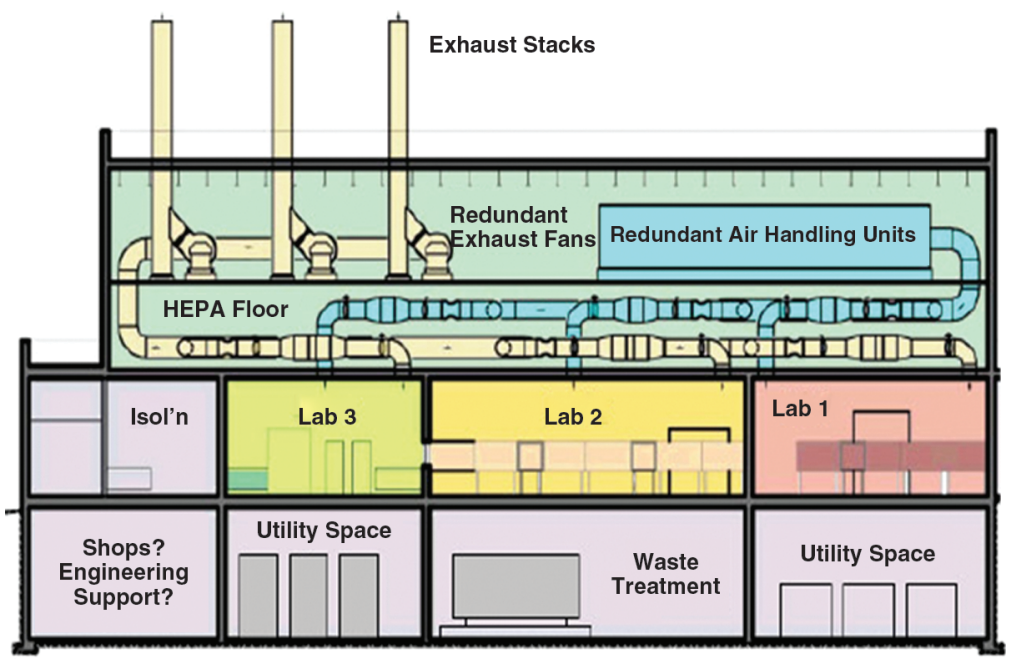

FIG. 1. Sample Receiving Facility cross section (FLAD). Samples would be processed and tested in three separate laboratories. These laboratories would be supported by extensive air filtration and waste-handling equipment, located above and below the laboratories, respectively. Color images available online at www.liebertonline.com/ast.

(11) Laboratory and engineering support facilities. Several types of facility support functions would need to be addressed, including cleaning and sterilization of equipment, instrumentation support and calibration, and provision of purified water and gases.

(12) Facility security system. When returned, the martian samples would be entities of singularly high value. Physical security to protect against theft, vandalism, and terrorism would be required.

(13) Other. To ensure containment and contamination control, the SRF would require operator safety systems such as fire protection, emergency oxygen supplies, an uninterruptable power supply, and other backup systems. The SRF would need to be operated under controlled conditions, including humidity, temperature, and potentially inert gas. The facility would need the capability to test and monitor all its essential safety systems and their backups. The facility must be able to maintain containment under a variety of off-nominal conditions, including power failure.

\section{B. FLAD team concept}

The FLAD team developed a preliminary concept of containment and identified the aspects of the preliminary concept that would require additional research before the concept could be developed further. They proposed a design that features three types of containment laboratories, each with unique functions. These laboratories would be located above utility and waste-treatment spaces and below air-handling spaces with roof exhausts. This design is similar to most BSL-4 labs currently operating in the United States (Fig. 1).

Laboratory 1 would be a glovebox ${ }^{2}$ facility, designed for manual initial processing of the spacecraft and samples.

\footnotetext{
${ }^{2}$ Class III Biological Safety Cabinets are sometimes called "gloveboxes." In this device, the interior of the cabinet is maintained under negative pressure (relative to the room), with HEPA filters attached to the supply and exhaust air systems. Class III BSCs can be connected together so that different activities can be performed sequentially on samples in containment. Autoclaves, incubators, dunk tanks, sample-transfer ports, and animal caging may be connected to the glovebox line.
}

Laboratory 2 would also be a glovebox facility that would use robots to perform initial sampling, subdivision, and sample testing. Gloves would be used only for maintenance and initial placement of equipment. Gloves would be sealed off during normal operations. Laboratory 3 would be a traditional BSL-4 suit facility, designed for biohazard and life detection, including small animal studies. All laboratories would be supplied with high efficiency particulate air (HEPA) filtered air, and each would have a separate combination of biological containment and air cleanliness appropriate to its operation.

The "gloveboxes" would be linked double-walled Class III Biological Safety Cabinets (BSCs), with reduced pressure between the walls (Fig. 2). This technology is designed to minimize contamination of both the laboratory and the samples in the event of a leak. The double-walled version of these cabinets and their associated gloves are not currently available (they are concept only), and represent significant design and operating challenges. While the single-walled versions are an accepted alternative to suits for BSL-4 operations, the double-wall technology would require detailed study to warrant such acceptance.

The FLAD SRF concept would include robots to perform initial sampling, subdivision, and sample testing. The current generation of industrial robots, particularly those used in microelectronics fabrication, has an impressive record of flexibility, reliability, and cleanliness. However, the capabilities of specific robots in the unique SRF environment must be the subject of extensive development, testing, and verification.

\section{C. LAS team concept}

The LAS team concept is a fully robotic SRF, with the exception of the in vivo biohazard testing. All sample handling, testing, and storage would be carried out inside one interconnected, multi-branching train of Class III BSCs (Fig. 3). The various branches would lead to different clusters. In the central node of each cluster, a robotic arm would perform the operations on the samples with specially designed end effectors, tools, instruments, and equipment. These robotic arms might have 6 degrees of freedom if needed for complex motions, or be a simpler type for moving samples from one station to another. 


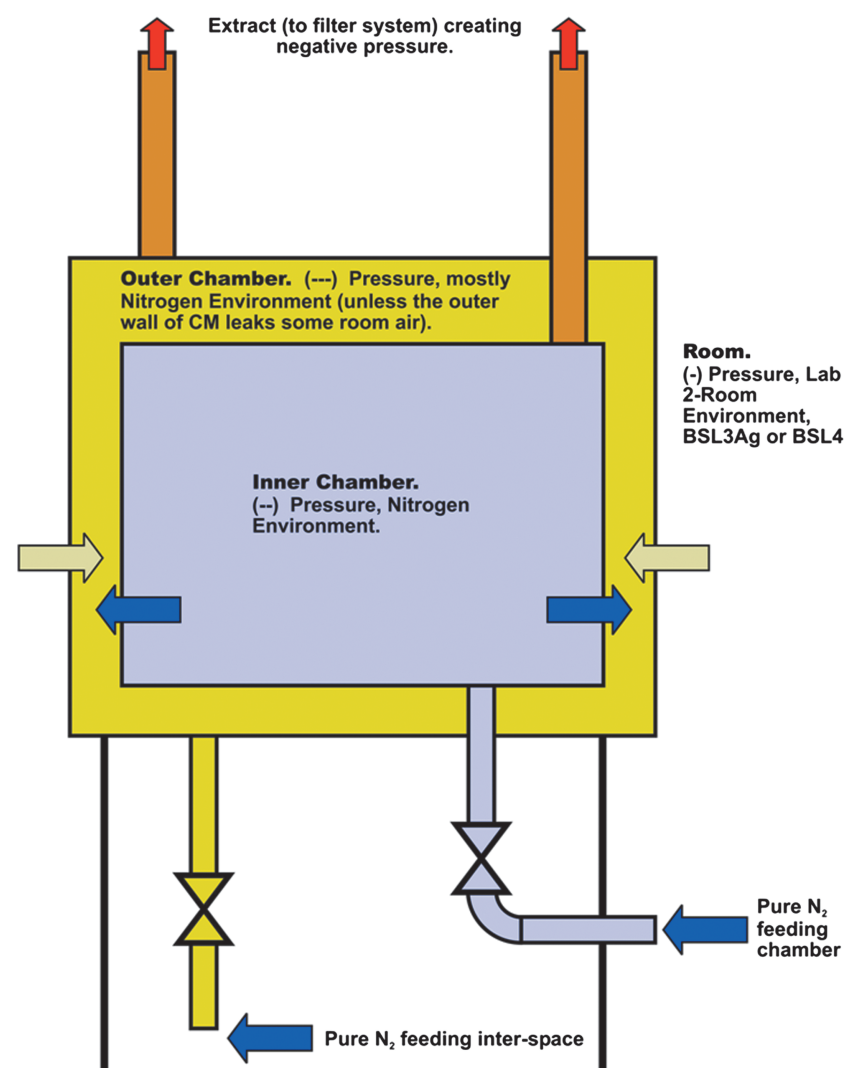

FIG. 2. Double-walled BSC schematic (FLAD). Samples would be stored and tested under nitrogen gas in the inner chamber, which would be held at negative pressure with respect to the surrounding room. Contamination from either the sample or the room would be captured in the outer chamber, which would be held at even lower pressure. Color images available online at www.liebertonline.com/ast.

A significant driver for the LAS team's SRF concept is the consideration that, based on past BSL- 4 experience, most breaches in containment are related to human error of one type or another and, in particular, issues associated with the gloves. In addition to susceptibility to needle punctures and other kinds of material failure, there are risks to the samples of imprecise manipulation of very small objects by doublegloved human hands. LAS proposed a design by which robotic manipulators would be employed, to the maximum extent possible, for direct contact with samples. Since multiple activities would need to be performed on the samples, this would require multiple isolator boxes and multiple robotic manipulators. LAS made the case that this approach would be the lowest-risk, both from the point of view of sample contamination and containment assurance, though it would clearly not be the lowest cost.

This concept would require a relatively small primary containment area that would use robotics to minimize human exposure and contamination. Biohazard testing would be carried out in a separate BSL-4 suit laboratory. These laboratories would be located above utility and wastetreatment spaces and below air-handling spaces.

The LAS team concept would use robots in most phases of laboratory operations, including spacecraft dismantling, removal of samples from their containers, initial sample characterization and subdivision, subsample packaging and transport, sample testing and analysis, and storage. Humans would perform hands-on maintenance and repair via gloveports. The capabilities of robots to perform all these tasks under stringent cleanliness and biosafety requirements would require extensive development, testing, and verification.

A unique feature of the LAS approach is the use of "common carriers" to transport and contain subsamples. The use of such devices is standard in the electronics fabrication industry, where conditions of extreme cleanliness are maintained in these "mini-environments" without requiring such stringent cleanliness in the entire laboratory.

The LAS team report also devotes considerable attention to the subjects of decontamination and sterilization. The SRF must have the capability to decontaminate and sterilize the entire laboratory, specific containment vessels, robots, and individual components. Sterilization techniques are well developed in industrial and research laboratories but would
FIG. 3. Fully robotic SRF floor plan (LAS) concept. Samples would be manipulated by robotic arms in an interconnected series of BSCs. Individual samples would be contained and transported in extremely clean "common carriers." Color images available online at www.liebertonline.com/ast.

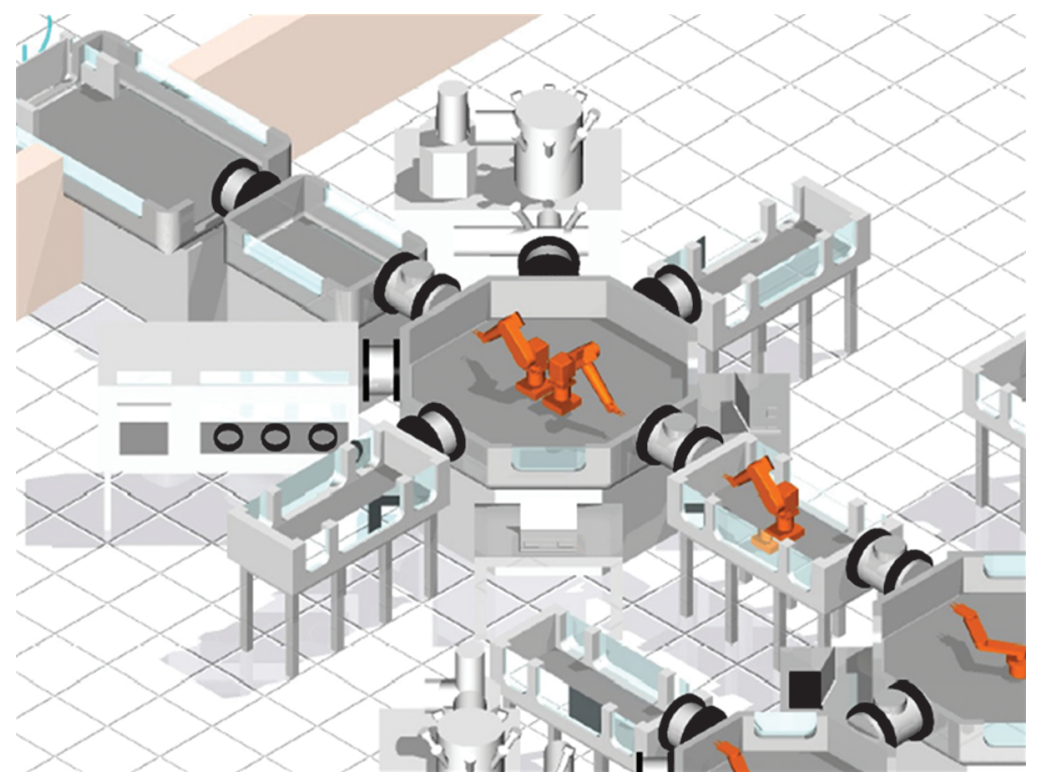




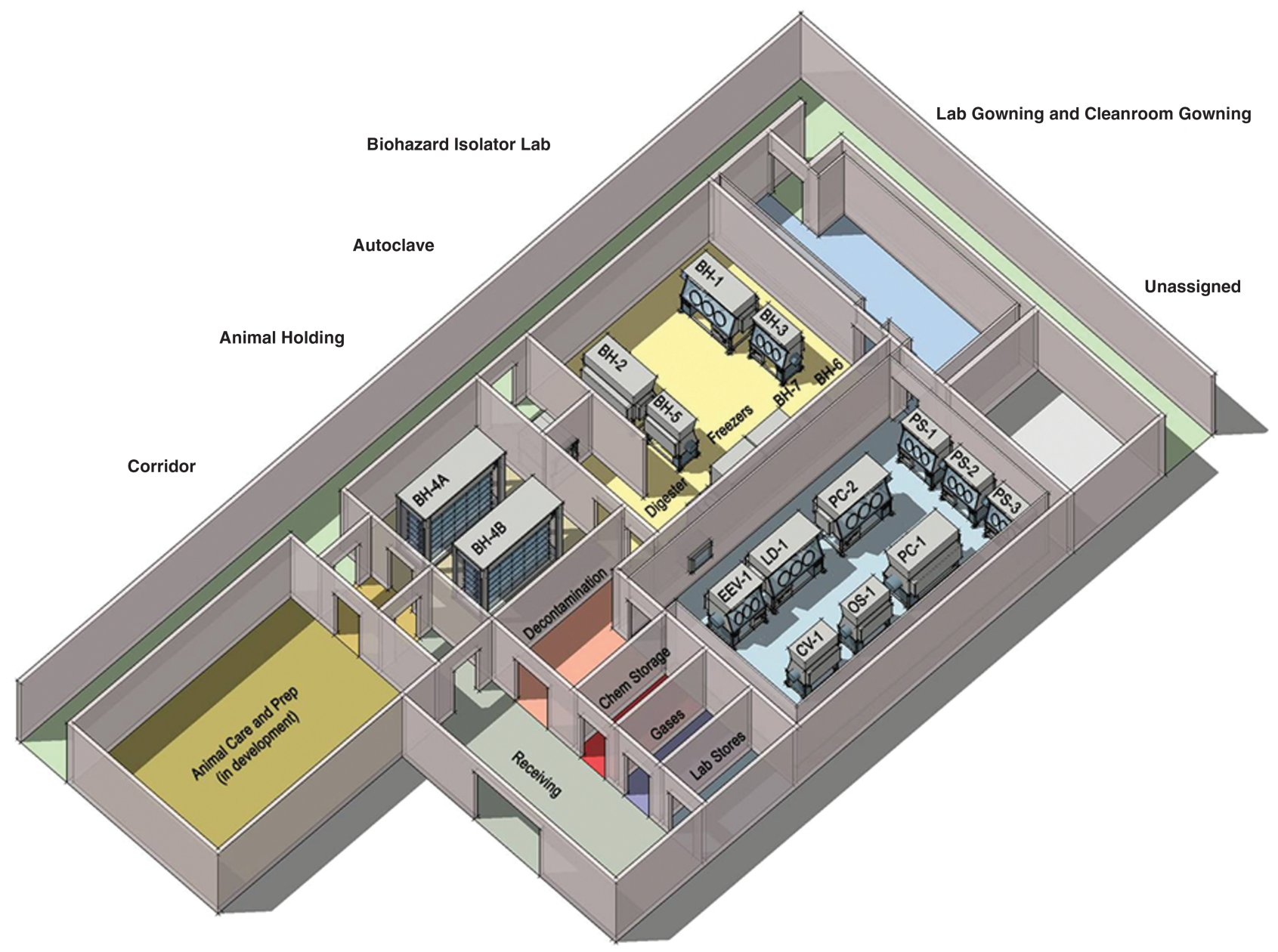

FIG. 4. Minimal robotic SRF floor plan (IDC). Samples would be stored and manipulated in individual glovebox isolators. Material would be moved between isolators via rapid-transfer ports. Color images available online at www.liebertonline.com/ast.

need to be adapted to the unique requirements of the SRF. That is, decontamination techniques (i.e., complete removal of organic and biological materials and contaminants) are not well developed, since, ordinarily, sterilization is deemed sufficient.

\section{D. IDC team concept}

The IDC team designed an SRF that would make minimal use of robotics technology. Their design features separate clean-room laboratories for physical and chemical testing, life detection, and biohazard testing (Fig. 4). Sample handling, physical and chemical testing, and storage would be conducted in separate, controlled-atmosphere "isolator" gloveboxes. ${ }^{3}$ The isolators would be maintained at negative pressure with respect to the laboratory, with HEPA filtered interiors for a Class 10 clean environment to protect the samples. The isolators would be located in BSL-3 level lab rooms that would allow the staff to be dressed in lab garments, rather than positive-pressure personnel suits.

\footnotetext{
3"Isolators" are not necessarily "gloveboxes." The term "isolator" is generally used to describe a bacteriology incubation device that may have gloves attached.
}

The isolators would not be interconnected, so each cabinet must be self-sufficient (i.e., with its own airlock, gas supplies, etc.). Samples would be moved between isolators by way of "rapid transfer ports." These containers would feature a double-door transfer system that would permit docking and undocking from isolators while maintaining biocontainment. Rapid transfer ports were originally developed for the nuclear industry but are now accepted for sample transport in high-level biosafety laboratories.

\section{E. Comparison of sample processing approaches}

In our comparison of results of the studies, we focus on the key characteristics associated with the sample processing functions, with minor discussion of facility maintenance and support staffing costs. The sample processing functions could be grouped into five steps, as outlined in Table 1, along with a summary of each team's approach. Any credible approach to each processing step must address both cleanliness and primary containment.

Containment approach. One method of containment common to the three industry studies is the use of Class III BSCs for primary containment in the initial sample processing steps. These cabinets would provide the highest level of 
Table 1. SRF Sample Processing Function and Team Approaches

\begin{tabular}{|c|c|c|c|c|}
\hline Step & Function & FLAD approach & LAS approach & IDC approach \\
\hline \multicolumn{2}{|c|}{$\begin{array}{l}\text { Summary approach to hardware } \\
\text { and sample flow }\end{array}$} & $\begin{array}{l}\text { Three-lab design with } \\
\text { mixture of robotic } \\
\text { and operator } \\
\text { manipulation of } \\
\text { samples }\end{array}$ & $\begin{array}{l}\text { Robotic-assisted } \\
\text { sample processing } \\
\text { in containment vessel } \\
\text { line plus BSL-4 in vivo } \\
\text { lab for biohazard test }\end{array}$ & $\begin{array}{l}\text { Large clean lab with } \\
\text { specialized } \\
\text { isolators; mainly } \\
\text { operator } \\
\text { manipulation of } \\
\text { samples }\end{array}$ \\
\hline 1 & $\begin{array}{l}\text { Receive all Earth- } \\
\text { return flight } \\
\text { hardware and } \\
\text { perform initial } \\
\text { disassembly }\end{array}$ & $\begin{array}{l}\text { Mobile containment } \\
\text { module mated to or } \\
\text { admitted through } \\
\text { airlock to } \\
\text { containment } \\
\text { module }(\mathrm{CM}) \text { in Lab } \\
\text { 1. Personnel in lab } \\
\text { garments would use } \\
\text { glove ports and } \\
\text { mechanical assists } \\
\text { in CM. }\end{array}$ & $\begin{array}{l}\text { Sealed mobile } \\
\text { containment module } \\
\text { would be moved into } \\
\text { CM using robotic } \\
\text { manipulation and } \\
\text { mechanical assists. } \\
\text { Truck dock room } \\
\text { provided in design. }\end{array}$ & $\begin{array}{l}\text { Upon arrival, Earth } \\
\text { Entry Vehicle } \\
\text { placed under } \\
\text { industrial hood, } \\
\text { cleaned by } \\
\text { personnel using } \\
\text { glove ports and } \\
\text { tools in BSL-3 attire, } \\
\text { then moved into } \\
\text { isolator in clean lab. }\end{array}$ \\
\hline 2 & $\begin{array}{l}\text { Receive sample } \\
\text { canister and } \\
\text { perform initial } \\
\text { disassembly and } \\
\text { sample inspection; } \\
\text { initiate subsampling } \\
\text { and physical testing }\end{array}$ & $\begin{array}{l}\text { Sample canister } \\
\text { transitions to Lab } 2 \\
\text { with higher controls } \\
\text { to protect samples. } \\
\text { Mainly robotic } \\
\text { manipulation in } \\
\text { CMs. Samples } \\
\text { transferred in } \\
\text { common carriers. }\end{array}$ & $\begin{array}{l}\text { Robotic manipulation } \\
\text { in specialized } \\
\text { characterization } \\
\text { clusters. Samples } \\
\text { transferred in } \\
\text { common carriers. }\end{array}$ & $\begin{array}{l}\text { Manipulation of } \\
\text { sample canister and } \\
\text { samples by } \\
\text { personnel in lab } \\
\text { garments using } \\
\text { glove ports and } \\
\text { tools in CM. } \\
\text { Physical testing in } \\
\text { Class } 10 \text { clean room. }\end{array}$ \\
\hline 3 & $\begin{array}{l}\text { Perform chemical and } \\
\text { life-detection testing } \\
\text { on samples }\end{array}$ & $\begin{array}{l}\text { Continued work in } \\
\text { Lab 2. Robotic } \\
\text { manipulation in } \\
\text { CMs. Samples } \\
\text { transferred in } \\
\text { common carriers. }\end{array}$ & $\begin{array}{l}\text { Robotic manipulation } \\
\text { in specialized } \\
\text { characterization } \\
\text { clusters. Samples } \\
\text { transferred in } \\
\text { common carriers. }\end{array}$ & $\begin{array}{l}\text { Manipulation of } \\
\text { samples by } \\
\text { personnel in lab } \\
\text { garments using } \\
\text { glove ports and } \\
\text { tools in CM. Class } \\
10 \text { clean room. }\end{array}$ \\
\hline 4 & $\begin{array}{l}\text { Perform biohazard } \\
\text { tests on samples }\end{array}$ & $\begin{array}{l}\text { Some continued work } \\
\text { in Lab 2. Remaining } \\
\text { life-detection and } \\
\text { biohazard testing in } \\
\text { Lab } 3 \text { or alternate } \\
\text { facility. Personnel in } \\
\text { BSL-4 class suits } \\
\text { work with animals, } \\
\text { plants, cultures, etc. }\end{array}$ & $\begin{array}{l}\text { Samples move through } \\
\text { airlock to } \\
\text { biocontainment area. } \\
\text { Personnel in BSL-4 } \\
\text { class suits work with } \\
\text { animals, plants, } \\
\text { cultures, etc. Use } \\
\text { biosafety cabinets } \\
\text { and gloveboxes. }\end{array}$ & $\begin{array}{l}\text { Manipulation of } \\
\text { samples by } \\
\text { personnel in lab } \\
\text { garments using } \\
\text { glove ports and } \\
\text { tools in CM. }\end{array}$ \\
\hline 5 & $\begin{array}{l}\text { Store samples in } \\
\text { pristine } \\
\text { environments }\end{array}$ & To be worked. & $\begin{array}{l}\text { Tested samples } \\
\text { retained at each } \\
\text { characterization } \\
\text { cluster for interim } \\
\text { storage. Remaining } \\
\text { samples kept in } \\
\text { pristine storage. }\end{array}$ & $\begin{array}{l}\text { Repetitive storage and } \\
\text { retrieval tasks use } \\
\text { telemanipulation in } \\
\text { isolators for } \\
\text { maximum } \\
\text { protection of } \\
\text { samples. }\end{array}$ \\
\hline
\end{tabular}

CM, containment module.

protection of the external environment from the internal contents. They are used in many industrial and biological research labs to provide the primary containment. They are of gas-tight construction but ventilated to provide critical environmental control, with both incoming and outgoing HEPA filtration.

The three industry study concepts also use other critical containment methods common in BSL-4 laboratories and facilities. They include careful designs of the lab space sur- rounding the BSCs, special waste treatment, air handling and utility systems, as well as safety and security provisions.

Cleanliness. All study concepts make use of many important cleanliness methods. For example, all three concepts propose the use of double-walled BSCs that, in addition to containment, would provide some protection for the samples. Also, in the initial process of dismantling the Earthreturn vehicle, all three concepts would clean and sterilize 
the outside of each spacecraft element and transfer the cleaned element(s) into the next pristine BSC.

Disparate sample sizes. All three concepts share some essential characteristics, such as their capability to deal with all forms of material likely to be contained in the returned sample. For example, all three SRF concepts plan to use appropriate forms of containers and tools/end effectors for gas, fines $(<1 \mathrm{~mm})$, coarse material $(>1 \mathrm{~mm})$, and "large rocks." The designs presented in the three study concepts also share the capability to capture the martian atmospheric gases, separate dust from this sample, and package each for testing and storage. They also have in common the notional capability to perform physical, chemical, life-detection, and biohazard testing on a portion of each solid sample.

The differences in the designs originate primarily from different ways to approach three issues:

(1) Approach to maintaining sample purity. The strategy of MSR would include bringing samples that might contain little or no evidence of life into an environment (an Earth laboratory) where sources of life-related contamination are essentially everywhere. Getting a laboratory "blank" down to as low a level as possible would likely take heroic efforts. Since human beings are a major potential source of biological contamination, it is important to carefully consider the proximity of humans to the martian samples.

(2) Approach to containment assurance. While the notion that any returned martian samples must be kept isolated is not unique, reduction of terrestrial contamination to an acceptable risk threshold could be established in a number of different ways.

(3) The relationship of instrumentation to the containment system. Would all the instruments required to carry out the purpose of the SRF need to be placed inside the isolator cabinets (the primary containment system), or could some of them be operated in an open laboratory? Some of the instruments that would be required by the test protocol might include relatively sophisticated devices, such as scanning electron microscopes or mass spectrometers.

\section{F. Building size, cost, and schedule}

At the heart of the motivation to conduct the SRF concept studies was the need to gain a better understanding of parameters relevant to program planning-facility scope or size, cost, and timing. The reports that were delivered, combined with some interpretation to enable direct comparison, provided insights in each key area.

(1) Building size. A key objective of this study was to estimate the minimum building size that would be sufficient to carry out the SRF function. The overall facility size estimated by three independent groups ranged from about 35,000 to 60,000 square feet. This floor space could be broken down as follows:

(a) 25,000-40,000 square feet of space related to the containment labs that constitute the facility core. This would include not just the contained laboratory rooms (which are estimated to be about 5,000-
10,000 square feet, depending on the implementation concept) but also the gowning area(s), decontamination showers, waste storage and treatment, interstitial space, clean sample storage, airshafts, and the perimeter corridor. The sample receiving requirements imply a receiving dock connected to a decontamination laboratory, which would need to be part of the containment core of the laboratory (one of its functions would be to test the spacecraft seals) - its space needs are estimated to be about 1,000 square feet for the decontamination lab and an additional 1-2 times that for the uncontained receiving dock and an associated support lab.

(b) 10,000-15,000 square feet of office, administrative, and logistical support space (including storage, security).

(c) $\sim 5,000$ square feet of high-containment labsupport space (including testing shops).

(2) Cost. Each of the three design teams estimated the cost and cost profile based on the SRF scenario that was presented to them and their interpretation of how to best implement it. At the end of the industry studies, the Mars Program study lead normalized the three different team estimates to place them on as comparable a footing as possible, and a composite budget profile was prepared (Table 2). The annual budgets in the composite are expressed in units of dollars for each respective fiscal year (so-called "real year" dollars), with an overall total of $\$ 121$ million. The permanent staff necessary to operate the SRF is estimated to be about 20-30 persons, for an annual operational cost of $\$ 7$ million. This estimate includes single-shift administrative, facility support and maintenance, and core

Table 2. Estimated Cost to Design, Build, AND OPERATE AN SRF

Assume MSR launches in 2013, samples arrive on Earth in 2016

\begin{tabular}{lcl}
\hline & $\begin{array}{c}\text { Annual budget } \\
\text { (millions, } \\
\text { real-year dollars) }\end{array}$ & \multicolumn{1}{c}{ Life-cycle phase } \\
\hline FY05 & 0.2 & $\begin{array}{c}\text { Oversight of advanced } \\
\text { technology development, } \\
\text { site selection }\end{array}$ \\
FY06 & 0.6 & Design \\
FY07 & 1 & \\
FY08 & 2 & Construction \\
FY09 & 3 & Commissioning \\
FY10 & 10 & Training, operational \\
FY11 & 26 & readiness testing \\
FY12 & 23 & Samples arrive, are active \\
FY13 & 22 & within facility \\
FY14 & 7 & \\
FY15 & 7 & \\
FY16 & 7 & \\
FY17 & 7 & \\
FY18 & 5 & \\
Total & $\mathbf{1 2 1}$ &
\end{tabular}

FY, fiscal year. 
building operations staff. These figures do not include the large number of personnel that would be required to carry out the work of the Test Protocol, such as guest scientists, planetary protection officials, and planetary program managers during the years of active sample handling and study within the SRF. Additionally, we caution that this cost estimate also does not include the research and technology development efforts necessary to provide key functionality in the SRF. Although this cost profile and total cost is specific to the study scenario, it might be adjusted to alternate future scenarios by applying the appropriate inflation factors. To make such a cost adjustment to alternative or future scenarios, it is crucial to refer to the key underlying assumptions concerning schedule and cost basis that were presented earlier in Section 3E.

(3) Schedule. The three teams each analyzed the schedule that would be needed to acquire permits, conduct design, construct the facility, and commission the SRF they envisioned. The estimated total schedule duration from the start of concept design and site selection activities to readiness to receive samples is approximately 7 years. Note that this estimate does not include the significant research and development effort required to reach the ready-to-build stage. Additionally, this schedule is estimated to vary by 1 to 2 years, primarily depending on whether NASA used an internal or external site selection process (an internal site selection process would mean that only properties under the jurisdiction of the Federal Government are considered, whereas external site selection would mean that private landowners, state governments, and institutions could also propose facility locations). The schedule would have to be lengthened by approximately 2 years should an external property purchase be required. For comparison, Rummel et al. (2002) and Atlas (2008) estimated that this amount of work would take 9 years and 10 years, respectively. An obvious difference is that these industry study teams were accustomed to designing and constructing buildings in an environment where things move more quickly than might be true of this unique SRF project.

\section{G. Areas for further analysis and technology development}

Analysis of the SRF design trade space has identified several major recognizable technology gaps [see also the planning reported by Mattingly et al. (2005), which was based on the same SRF studies reported in this paper] and some areas requiring further analysis.

Decontamination techniques. Methods for removing organic, inorganic, and particulate contamination from containers, BSCs, robotic manipulators, and hand tools coming into contact with the samples or their environment.

Double-walled glovebox containment. Design, fabrication, testing, and certification of a biosafety cabinet with a double wall enclosing a low-pressure space, designed to trap leakage from the samples as well as contaminants from the outside laboratory.

Robotics. Demonstrate operations of dexterous ultra-clean robots capable of sample transport and sample manipulation (see also Mani et al., 2008). As pointed out by Bell and Allen (2005), a key issue is whether the robotics can be made compatible with the cleanliness requirements.

Pristine sample preservation. Methods of preserving martian rock, soil, and atmosphere samples for long-term storage and distribution to researchers in a wide variety of disciplines.

Scientific equipment required for proper sample analysis. Specific instruments to conduct the testing required for assessment of possible life and biohazards in martian rock, soil, and atmosphere samples.

\section{Discussion and Future Planning}

Three independent industry studies produced complementary high-level concepts for an SRF dedicated to an MSR mission. These industry study concepts represent a "snapshot in time" relative to our understanding of the characteristics of martian samples, the nature of a sample return mission, the requirements of planetary protection, and the role of international collaboration and regulations in the mission. These detailed studies will need to be redone at some point in the future in light of our updated knowledge of Mars, an updated Test Protocol (note the emphasis on this in Atlas, 2008), the possible internationalization of MSR (e.g., iMARS, 2008; Mani et al., 2008), and our evolving budgetary and political realities.

\section{A. Some considerations affecting SRF size and cost}

The cost presented above is based on a specific scenario, with a circumscribed set of assumptions and requirements. However, the size and cost of the SRF is sensitive to the several possible changes in the assumptions and requirements, some of which might have significant effect on its cost.

Partnering options. The SRF scenario evaluated in this study was assumed to be a single, new, stand-alone building. Locating this building as part of a campus of other buildings could enable the sharing or avoidance of certain costs, such as perimeter security, access to transportation and utilities, access to a trained technician pool, avoidance of legally protected areas, and so forth. Although the general potential for cost saving is obvious, this cannot be evaluated in detail without specific designs.

Making use of existing construction. A single, new, stand-alone building is the simplest (and perhaps the only) way to meet the expected cleanliness requirements. However, it may be possible to configure the SRF as a newly constructed wing of an existing building, or perhaps even as a retrofit of an existing building.

Live animal testing. Because the present Draft Test Protocol (Rummel et al 2002) calls for conventional wholeorganism animal and plant in vivo testing, a significant part of the floor space of the containment laboratory core in the three scenarios relates to animal holding and evaluation areas. Since the Draft Test Protocol was published in 2002 and used as a key element of the baseline requirements for the 2004 industry study concepts, the fields of microbiology and biohazard detection have advanced markedly. The limits of detection and classification of microbial life in 
environmental samples have improved significantly, and the use of animals in biohazard testing has been increasingly replaced by cellular and molecular analyses. If a future version of the test protocol eliminates this requirement in accordance with state-of-the-art practices and refinements at the time the final protocol is implemented, the SRF design would potentially be simpler.

More than one SRF. The study teams each analyzed the design, construction, and operation of a single SRF. However, in the future it might be possible or required that there are two or more SRFs in different locations, possibly in different countries. If there are multiple SRFs, they could conceivably either be designed with identical capabilities or, alternatively, with the capability to perform different, complementary tests. The possibility of a two-SRF scenario has been discussed both within the 2003-2005 MSR Project and by iMARS (2008) as a potentially desirable, or perhaps even politically necessary, attribute of an international MSR mission. A specific variation on the single-SRF approach (alluded to by Rummel et al 2002 in the Draft Test Protocol) is the possibility that live animal testing could be done in a secondary facility that meets the biocontainment requirements of the SRF, which would thus simplify the design and cost of the primary facility. A prerequisite for any two-SRF scenario would be a means by which to move unsterilized samples between facilities and the approval to do so.

SRF expandability. This study evaluated the minimum possible SRF that would be required to execute the test protocol. If the samples are determined to be non-hazardous (or are rendered non-hazardous by sterilization), they would be made available for allocation to the world's research community. However, if signs of life are detected in one or more samples, it might become necessary for future study of the returned samples to take place in ongoing containment. The samples would then need to be evaluated with additional methods beyond those used in the Draft Test Protocol. The SRF may need to be designed so that it could be expanded to accommodate additional laboratories; if this functional requirement were accepted, it could have significant cost implications.

\section{Conclusions}

Based on the analysis and interpretation of the 2004 industry study concepts, we suggest the following considerations for planning an SRF to meet the needs of a future MSR mission.

(1) Design. There is more than one possible design for a stand-alone SRF that would meet the requirements of MSR. Because the full set of requirements is not defined at this time, it is not possible to optimize the design. However, it is possible to understand the likely possibilities enough to generate first-order budgeting/ planning parameters.

(2) Size. A minimal stand-alone SRF is estimated to have an overall size of about 35,000-60,000 square feet, including 25,000-40,000 square feet of containmentrelated space that makes up the facility core (of which 5,000-10,000 square feet are the high-containment laboratory rooms; the remainder would consist of facility support systems including air handling, chemical showers, waste cookers, etc.), up to 5,000 square feet of high-containment lab support space (test and repair shops), and 10,000-15,000 square feet of office, administrative, and logistical support space (including storage and security).

(3) Schedule. The schedule needed to construct and commission an SRF is estimated to be 7-9 years. Most of this variance relates to whether NASA uses an internal or an external site selection process. Because of the schedule-constrained nature of an MSR (i.e., once the samples have left Mars, spacecraft trajectories have a defined schedule that cannot be easily slipped), it is prudent to add additional schedule reserves, and a good planning number is 10 years.

(4) Capital cost. The cost of an SRF would depend upon the specific design approach, as well as on the final test protocol executed in the facility. However, for future planning a reasonable budget estimate is the escalated equivalent of $\$ 120$ million real-year dollars, using the 2013 MSR scenario described in Table 2.

(5) Operating cost. During the years the martian samples would be evaluated in the facility, the annual building operating budget would be estimated at $\$ 7$ million, which includes a building operations staff of 20-30 persons. This estimate does not include the large number of personnel that would be required to carry out the work of the Test Protocol.

(6) Advance technology development. Most of the technology needed to design and construct an SRF already exists in the biosafety, pharmaceutical, and sample curation communities. However, along with decontamination techniques, double-walled glovebox containment, dexterous ultra-clean robotics, pristine sample preservation, and scientific equipment required for proper sample analysis, some aspects of the project will need to be planned at two specific points in the building life cycle: (a) those that affect the facility design and will need to be known before facility construction, (b) those that affect the instrumentation, the experiments, or both, that will need to be known before the facility is equipped.

(7) Partnering opportunities. Although not fully analyzed in the industry study concepts, it is likely that partnering opportunities might result in cost savings, operational efficiency, or other benefits. Such opportunities could be evaluated against the reference planning parameters described above to determine whether this would be a better way to meet the needs of an MSR than with a stand-alone SRF.

\section{Acknowledgments}

The authors wish to recognize the teams and dedicated individuals at Industrial Design and Construction (IDC) of Portland, Oregon; Lord, Aeck, Sargent (LAS) of Atlanta, Georgia; and Flad \& Associates (FLAD) of Madison, Wisconsin, for their diligence, interest, and hard work that comprises the core of this paper. We also want to acknowledge study participant and reviewer Jonathan Richmond, and members of the 1999-2002 Mars Returned Sample Handling team Lisa Fletcher, Mary Sue Bell, Judy Allton, 
Stan Farkas, Geoff Briggs, Laurie Carrillo, Kimberly Cyr, Sandy Dawson, Janis Graham, Ragnhold Landheim, Rocco Mancinelli, Ed Mickelson, and Nancy Robertson, whose work led up to the SRF design studies. Thanks also to Margaret Race, John Rummel, and Joe Parrish who all contributed to our understanding during the planning and performance of the SRF design study effort; Jeff Schanz, who provided continued technical assistance after the delivery of the studies; and reviewer Richard Mattingly. The research described in this paper carried out at the Jet Propulsion Laboratory, California Institute of Technology, was done so under a contract with the National Aeronautics and Space Administration.

\section{Author Disclosure Statement}

No competing financial interests exist. The cost information contained in this document is of a budgetary and planning nature and is intended for informational purposes only. It does not constitute a commitment on the part of JPL and/or Caltech.

\section{Abbreviations}

BSC, Biosafety Cabinet or Biological Safety Cabinet; BSL, Biosafety Level; CAPTEM, Curation and Analysis Planning Team for Extraterrestrial Materials; FLAD, Flad \& Associates; HEPA, high efficiency particulate air; IDC, Industrial Design and Construction; LAS, Lord, Aeck, Sargent; LRL, Lunar Receiving Laboratory; MSHARP, Mars Sample Handing and Requirements Panel; MSR, Mars Sample Return; NEPA, the National Environmental Policy Act; NRC, National Research Council; SRF, Sample Receiving Facility.

\section{References}

Allton, J.H., Bagby, J.R., and Stabekis, P.D. (1998) Lessons learned during Apollo lunar sample quarantine and sample curation. Adv. Space Res. 22:373-382.

Atlas, R. (2008) Mars Sample Return Receiving Facility. Presentation to the Planetary Science Subcommittee. Available online at http://www.lpi.usra.edu/pss/presentations/ 200803/04-Atlas-PPSonMSR.pdf.

Backes, P., Onstott, T., Bar-Cohen, Y., Badescu, M., Pratt, L., Helmick, D., Sherrit, S., Johnson, A., and Bao, X. (2009) Planetary sample sealing for caching. In Proceedings of the 2009 IEEE Aerospace Conference, Institute of Electrical and Electronics Engineers, Piscataway, NJ.

Bar-Cohen, Y., Wu, J., Bley, S., Olorunsola, A.K., Rivellini, T.P., Wincentsen, J., and Gershman, R. (2005) Simultaneous separation, seaming and sealing using brazing (S3B) for sample containerization and planetary protection [Paper \#\#5762-43]. In Proceeding of the Industrial and Commercial Applications of Smart Structures Technologies, SPIE Smart Structures and Materials Symposium, The International Society for Optical Engineering, Bellingham, WA.

Bell, M.S. and Allen, C. (2005) Cleanroom robotics-appropriate technology for a sample receiving facility [abstract 1395]. In $36^{\text {th }}$ Lunar and Planetary Science Conference, Lunar and Planetary Institute, Houston. Available online at http://www. lpi.usra.edu/meetings/lpsc2005/pdf/1395.pdf.

Carr, M.H., editor. (1999) Mars Sample Handling and Requirements Panel (MSHARP) Final Report [NASA TM-1999-209145], NASA Jet Propulsion Lab, Pasadena, California.
CDC/NIH. (2007) Biosafety in Microbiological and Biomedical Laboratories $(B M B L), 5^{\text {th }}$ ed., U.S. Department of Health and Human Services Centers for Disease Control and Prevention and National Institutes of Health, Washington, DC. Available online at http://www.cdc.gov/od/ohs/biosfty/bmbl5/bmbl5toc .htm.

COSPAR (2008) COSPAR Planetary Protection Policy, Committee on Space Research of the International Council for Science, Paris. Available online at: http://cosparhq.cnes.fr/Scistr/ PPPolicy(20-July-08).pdf.

DeVincenzi, D.L., Bagby, J., Race, M., and Rummel, J.D., editors. (1999) Mars Sample Quarantine Protocol Workshop [NASA CP1999-208772], NASA Ames Research Center, Moffett Field, California.

Gershman, R., Adams, M., Dillman, R., and Fragola, J. (2005) Planetary protection technology for Mars Sample Return [Paper \#2.1607]. In Proceedings of the 2005 IEEE Aerospace Conference, Institute of Electrical and Electronics Engineers, Piscataway, NJ. iMARS. (2008) Preliminary Planning for an International Mars Sample Return Mission: Report of the International Mars Architecture for the Return of Samples (iMARS) Working Group, International Mars Architecture for the Return of Samples Working Group. Available online at http://mepag.jpl.nasa .gov/reports/iMARS_FinalReport.pdf.

Mangus, S. and Larsen, W. (2004) Lunar Receiving Laboratory Project History [NASA/CR-2004-208-938], NASA, Washington DC. Available online at http://ston.jsc.nasa.gov/collections/ TRS/_techrep/CR-2004-208938.pdf.

Mani, P., Nelson, B., Pauli, U., Kray, R., Huntley, P., Ross, F., Heuer, M., and Hofmann, B.A. (2008) Preliminary concept for a Mars Sample Receiving Facility [abstract \# PPP2-000608]. In COSPAR 2008, Committee on Space Research of the International Council for Science, Paris. Available online at http://www.cospar-assembly.org/abstractcd/COSPAR-08/ abstracts/data/pdf/abstracts/PPP2-0006-08.pdf.

Mattingly, R., Hayati, S., and Udomkesmalee, G. (2005) Technology development plans for the Mars Sample Return mission [Paper \#151]. In Proceedings of the 2005 IEEE Aerospace Conference, Institute of Electrical and Electronics Engineers, Piscataway, NJ.

Moura, D., Jordan, F., Pradier, A., May, L., Santovincenzo, A., Mattingly, R., Kerridge, S., Khan, M., Gardini, B., and McCuistion, D. (2008) The road to an international architecture for Mars Sample Return: The iMARS Team view [IAC08-A3.1.3]. In The 59 ${ }^{\text {th }}$ International Astronautical Congress, International Astronautical Federation, Paris.

MEPAG ND-SAG. (2008) Science priorities for Mars Sample Return. Astrobiology 8:489-535. Available online at http:// mepag.jpl.nasa.gov/reports/index.html.

NRC. (1978) Strategy for the Exploration of the Inner Planets: 19771987, National Academy Press, Washington DC.

NRC. (1990a) Update to Strategy for Exploration of the Inner Planets, National Academy Press, Washington DC.

NRC. (1990b) International Cooperation for Mars Exploration and Sample Return, National Academy Press, Washington DC.

NRC. (1994) An Integrated Strategy for the Planetary Sciences: 1995-2010, National Academy Press, Washington DC.

NRC. (1996) Review of NASA's Planned Mars Program, National Academy Press, Washington DC.

NRC. (1997) Mars Sample Return Issues and Recommendations, Task Group on Issues in Sample Return, Space Studies Board, Commission on Physical Sciences, Mathematics, and Applications, National Research Council, National Academy Press, Washington DC. 
NRC. (2002) The Quarantine and Certification of Martian Samples, National Academy Press, Washington DC.

NRC. (2007) An Astrobiology Strategy for the Exploration of Mars, The National Academies Press, Washington DC.

Neal, C.R. (2000) Issues involved in a martian sample return: integrity preservation and the Curation and Analysis Planning Team for Extraterrestrial Materials (CAPTEM) position. J. Geophys. Res. 105:22487-22506.

O'Neil, W.J. and Cazaux, C. (2000) The Mars Sample Return project. Acta Astronaut. 47:453-465.

Race, M.S. and Hammond, E. (2008) An evaluation of the role and effectiveness of institutional biosafety committees in providing oversight and security at biocontainment laboratories. Biosecurity and Bioterrorism: Biodefense Strategy, Practice and Science 6:19-35.

Race, M.S. and Rummel, J.D., editors. (2000) Mars Sample Handling Protocol Workshop Series. Interim Report of the Workshop Series: Workshop 1 Proceedings and Final Report [NASA/ CP-2000-209624], NASA, Washington DC.

Race, M.S., Kovacs, G.T.A., Rummel, J.D., and Acevedo, S.E., editors. (2001a) Mars Sample Handling Protocol Workshop Series. Interim Report of the Workshop Series: Workshop 2 Proceedings and Final Report [NASA/CP 2001-210923], NASA, Washington DC.

Race, M.S., Nealson, K., Rummel, J.D., and Acevedo, S.E., editors. (2001b) Mars Sample Handling Protocol Workshop Series. Interim Report of the Workshop Series: Workshop 3 Proceedings and
Final Report [NASA/CP-2001-211388], NASA, Washington DC.

Rummel, J.D., Race, M.S., DeVincenzi, D.L., Schad, P.J., Stabekis, P.D., Viso, M., and Acevedo, S.E., editors. (2002) A Draft Test Protocol for Detecting Possible Biohazards in Martian Samples Returned to Earth [NASA/CP-20-02-211842], NASA Ames Research Center, Moffett Field, CA.

Stephenson, D.D. and Willenberg, H.J. (2006) Mars ascent vehicle key elements of a Mars Sample Return mission. In 2006 IEEE Aerospace Conference, Institute of Electrical and Electronics Engineers, Piscataway, NJ.

U.S. Department of Health and Human Services (2007) Biosafety in Microbiological and Biomedical Laboratories, $5^{\text {th }}$ ed., US Government Printing Office, Washington DC. Available onlie at http://www.cdc.gov/od/ohs/biosfty/bmbl5/bmbl5toc.htm.

Address correspondence to: Deborah S. Bass

Mars Program Office Jet Propulsion Laboratory California Institute of Technology 4800 Oak Grove Drive Pasadena, CA 91109-8099

E-mail: Deborah.Bass@jpl.nasa.gov 